\title{
Correction to: Effects of noise on mental performance and annoyance considering task difficulty level and tone components of noise
}

\author{
Mohammad Javad Jafari $^{1,2} \cdot$ Marzieh Sadeghian $^{1,2} \cdot$ Ali Khavanin $^{3} \cdot$ Soheila Khodakarim $^{4} \cdot$ Amir Salar Jafarpisheh $^{5}$
}

Published online: 25 June 2019

(C) Springer Nature Switzerland AG 2019

\section{Correction to: J Environ Health Sci Eng}

https://doi.org/10.1007/s40201-019-00353-2

The correct first names and last names are "Mohammad Javad Jafari”, "Marzieh Sadeghian”, “Ali Khavanin”, "Soheila Khodakarim”, “Amir Salar Jafarpisheh".

The original article has been corrected.

Publisher's note Springer Nature remains neutral with regard to jurisdictional claims in published maps and institutional affiliations.

The online version of the original article can be found at https://oi.org/ 10.1007/s40201-019-00353-2

Marzieh Sadeghian

mrz.sadeghian@gmai.com

1 Environmental and Occupational Hazards Control Research Center, Shahid Beheshti University of Medical Sciences, Tehran, Iran

2 Department of Occupational Health Engineering, School of Public Health and Safety, Shahid Beheshti University of Medical Sciences, Tehran, Iran

3 Department of Environmental and Occupational Health Engineering, Tarbiat Modares University, Tehran, Iran

4 Department of Epidemiology, School of Public Health and Safety, Shahid Beheshti University of Medical Sciences, Tehran, Iran

5 Department of Ergonomics, University of Social Welfare and Rehabilitation Sciences, Tehran, Iran 